\title{
Light Baryons in a Constituent Quark Model with Chiral Dynamics
}

\author{
L. Ya. Glozman ${ }^{1}$, Z. Papp ${ }^{2}$, W. Plessas ${ }^{1}$ \\ ${ }^{1}$ Institute for Theoretical Physics, University of Graz, \\ Universitätsplatz 5, A-8010 Graz, Austria \\ ${ }^{2}$ Institute of Nuclear Research of the Hungarian Academy of Sciences, \\ Bem tér 18/c, P.O. Box 51, H-4001 Debrecen, Hungary
}

\begin{abstract}
It is shown from rigorous three-body Faddeev calculations that the masses of all 14 lowest states in the $N$ and $\Delta$ spectra can be described within a constituent quark model with a Goldstone-boson-exchange interaction plus linear confinement between the constituent quarks.
\end{abstract}

PACS numbers 12.39.-x, 12.38.Lg, 12. 40.Yx, 14.20.Gk

Submitted to Physics Letters B

Preprint UNIGRAZ-UTP 27-01-96 
Recently it has been suggested [1, 2] that beyond the scale of spontaneous breaking of chiral symmetry $(S B \chi S)$ light and strange baryons can be viewed as systems of three constituent quarks which interact by the exchange of Goldstone bosons (pseudoscalar mesons) and are subject to confinement. This concept is motivated by the idea that instead of operating with the original QCD degrees of freedom, i.e. current quarks and gluon fields, it is appropriate, in low-energy QCD, to deal with constituent quarks and chiral boson fields. This is in close analogy to solid-state physics where instead of considering original degrees of freedom (light electrons, a lattice of ions and the electromagnetic field) one works with "heavy" electrons with dynamical mass, phonons and their interactions.

In QCD the approximate $S U(3)_{L} \times S U(3)_{R}$ chiral symmetry in the $(\mathrm{u}, \mathrm{d}, \mathrm{s})$ sector is realized in the hidden Nambu-Goldstone mode at low temperature and density, i.e. is spontaneously broken up to $S U(3)_{V}$. This is manifested in the existence of the octet of low-mass pseudoscalar mesons representing approximate Goldstone bosons. The flavor singlet $\eta^{\prime}$ gets decoupled from the original $U(3)$ nonet due to instanton fluctuations in the QCD vacuum [3]. With respect to the fermion fields, the effect of $S B \chi S$ is that the valence current quarks acquire a dynamical mass 4 , 5]. As it was suggested within a microscopical approach [6], this occurs when quarks propagate through instantons in the QCD vacuum [7]. Thus in low-energy QCD one may rely on a chiral Lagrangian with dynamical fermion fields (constituent quarks) and nine chiral fields. Of course, the full effective chiral Lagrangian is not known. However, any possible (ps or pv) coupling of the constituent quarks and chiral meson fields will imply a Goldstone-boson exchange (GBE) interaction between the constituent quarks when the chiral meson fields are integrated out of the Fock space.

Already in refs. [1, 2] it has been indicated that this GBE interaction will be capable of describing all features of the light and strange baryon spectra. In particular, 
it has been shown how the spin-flavor symmetry of the GBE can resolve some longstanding problems in baryon spectroscopy. By taking into account the GBE within first-order perturbation theory one could explain (i) the correct level ordering of the positive- and negative-parity states in light and strange baryon spectra as well as the approximate splittings in those spectra and (ii) the branching ratios of $N^{*} \rightarrow N \eta$, $\Lambda^{*} \rightarrow \Lambda \eta$ and $\Sigma^{*} \rightarrow \Sigma \eta$ decays $[8]$.

In the present work we report results for the light baryon spectra obtained from rigorous three-body Faddeev calculations. Besides the confinement potential, which is here taken in linear form, the GBE interaction between the constituent quarks is taken into account to all orders. Our results further support the adequacy of the GBE for baryon spectroscopy. In particular, we shall demonstrate the description of all 14 lowest states in the $N$ and $\Delta$ spectra in agreement with phenomenology.

For the GBE the spin-spin component of the interaction between the constituent quarks $i$ and $j$ consists of the octet and singlet exchange components:

$$
\begin{gathered}
V_{\chi}^{\text {octet }}\left(\vec{r}_{i j}\right)=\left\{\sum_{a=1}^{3} V_{\pi}\left(\vec{r}_{i j}\right) \lambda_{i}^{a} \lambda_{j}^{a}+\sum_{a=4}^{7} V_{K}\left(\vec{r}_{i j}\right) \lambda_{i}^{a} \lambda_{j}^{a}+V_{\eta}\left(\vec{r}_{i j}\right) \lambda_{i}^{8} \lambda_{j}^{8}\right\} \vec{\sigma}_{i} \cdot \vec{\sigma}_{j}, \\
V_{\chi}^{\text {singlet }}\left(\vec{r}_{i j}\right)=\frac{2}{3} \vec{\sigma}_{i} \cdot \vec{\sigma}_{j} V_{\eta^{\prime}}\left(\vec{r}_{i j}\right) .
\end{gathered}
$$

Here $\vec{\sigma}$ and $\vec{\lambda}$ are the quark spin and flavor matrices, respectively. In the simplest case, when the boson field satisfies the linear Klein-Gordon equation, one has the following spatial dependence for the meson-exchange potentials in (1) and (2):

$$
\begin{gathered}
V_{\gamma}\left(\vec{r}_{i j}\right)=\frac{g_{\gamma}^{2}}{4 \pi} \frac{1}{3} \frac{1}{4 m_{i} m_{j}}\left\{\mu_{\gamma}^{2} \frac{e^{-\mu_{\gamma} r_{i j}}}{r_{i j}}-4 \pi \delta\left(\vec{r}_{i j}\right)\right\} \\
\left(\gamma=\pi, K, \eta, \eta^{\prime}\right),
\end{gathered}
$$


with quark and meson masses $m_{i}$ and $\mu_{\gamma}$, respectively. We refer to paper [2] for a detailed description of the properties of the octet-exchange interaction (1) within and beyond the $S U(3)$ limit.

Eq. (3) contains both the traditional long-range Yukawa potential as well as a $\delta$-function term. It is the latter that is of crucial importance for baryon physics. It is strictly valid only for pointlike particles. It must be smeared out, however, as the constituent quarks and pseudoscalar mesons have in fact finite size. Further it is quite natural to assume that at distances $r \ll r_{0}$, where $r_{0}$ can be related to the constituent quark and pseudoscalar meson sizes, there is no chiral boson-exchange interaction as this is the region of perturbative QCD with original QCD degrees of freedom. The interactions at these very short distances are not essential for the lowenergy properties of baryons. Consequently we use a two-parameter representation for the $\delta$-function term in $(3)$

$$
4 \pi \delta\left(\vec{r}_{i j}\right) \Rightarrow \frac{4}{\sqrt{\pi}} \alpha^{3} \exp \left(-\alpha^{2}\left(r-r_{0}\right)^{2}\right) .
$$

Obviously this substitution is exact in the limit $r_{0} \rightarrow 0, \alpha \rightarrow \infty$. Following the arguments above one should also cut off the Yukawa part of the GBE for $r<r_{0}$. In order to avoid any cut-off parameter we use a step-function cut-off at $r=r_{0}$. It may be introduced by the analytically smooth representation $\left[1-\left(1+\exp \left(\beta\left(r-r_{0}\right)\right)\right)^{-1}\right]^{5}$ with $\beta \rightarrow \infty$. We have tested that increasing $\beta$ beyond the actually employed value of $\beta=20 \mathrm{fm}^{-1}$ does not change the results noticeably. For the meson masses in (3) we take their physical values, $\mu_{\pi}=139 \mathrm{MeV}, \mu_{\eta}=547 \mathrm{MeV}, \mu_{\eta^{\prime}}=958 \mathrm{MeV}$, and for the $\mathrm{u}$ and $\mathrm{d}$ constituent quarks we use $m=340 \mathrm{MeV}$, like it is suggested from nucleon magnetic moments.

The $\pi q$ coupling constant can be extracted from the phenomenological pionnucleon coupling [2] as $\frac{g_{8}^{2}}{4 \pi}=0.67$. For simplicity (and to avoid any additional free 
parameter) the same coupling constant is assumed for the coupling between the $\eta$ meson and the constituent quark. This is exactly in the spirit of unbroken $S U(3)_{F}$ symmetry. For the flavor-singlet $\eta^{\prime}$, however, we must take a different coupling $\frac{g_{0}^{2}}{4 \pi}$, as the $U(3)_{V}$ symmetry is known to be very strongly broken to $S U(3)_{V} \times U(1)_{V}$. This fact is best illustrated by the failure of the Gell-Mann-Oakes-Renner relations [9] for the flavor singlet [10]. Lacking a phenomenological value, we treat $g_{0}^{2} / 4 \pi$ as a free parameter.

In the present calculation we neglect tensor meson-exchange forces. We expect their role to be of minor importance for the main features of baryon spectra [2] (mainly due to the absence of the strong $\delta$ - function part in this case). We emphasize that the primary aim in this paper is to demonstrate the feasibility and advantages of the GBE model in baryon spectroscopy. For this purpose the dynamical ingredients of (1) and (2) are already sufficient.

Our full interquark potential is thus given by

$$
V\left(\vec{r}_{i j}\right)=V_{\chi}^{\text {octet }}\left(\vec{r}_{i j}\right)+V_{\chi}^{\text {singlet }}\left(\vec{r}_{i j}\right)+C r_{i j} .
$$

While all masses and the octet coupling constant are predetermined, we treated $r_{0}, \alpha,\left(g_{0} / g_{8}\right)^{2}$, and $C$ as free parameters and determined their values to be:

$$
r_{0}=0.43 f m, \alpha=2.91 \mathrm{fm}^{-1},\left(g_{0} / g_{8}\right)^{2}=1.8, C=0.474 \mathrm{fm}^{-2} .
$$

Notice that we do not need any constant $V_{0}$, which is usually added to the confining potential. In fact only four free parameters suffice to describe all 14 lowest states of the $N$ and $\Delta$ spectra, including the absolute value of the nucleon (ground state).

The quark-quark potential (5) constitutes the dynamical input into our 3-body Faddeev calculations of the baryon spectra. The Faddeev equations were solved along 
the new method of ref. [11, designed for an efficient solution of any 3-body bound state problem. It has already been successfully employed in atomic and nuclear problems before. We have carefully checked the accuracy of the results for all baryon levels, in particular we have ensured perfect convergence with respect to subsystem angular-momentum states included 凹.

We show our results in Table 1 and Fig. 1 for the parametrization of the qq interaction given above. It is well seen that the whole set of lowest $N$ and $\Delta$ states is quite correctly reproduced. In the most unfavourable cases deviations from the experimental values do not exceed $3 \%$ ! In addition all level orderings are correct. In particular, the positive-parity state $N(1440)$ (Roper resonance) lies below the pair of negative-parity states $N(1535)-N(1520)$. The same is true in the $\Delta$ spectrum with $\Delta(1600)$ and the pair $\Delta(1620)-\Delta(1700)$. Thus a long-standing problem of baryon spectroscopy is now definitely resolved. We emphasize again that the qq potential (5) is able to predict also the absolute value of the nucleon mass. In previous models an arbitrary constant was usually needed to achieve the correct value of $939 \mathrm{MeV}$.

At the present stage of our investigation of the baryon spectra with the GBE interaction we have left out the tensor forces. Therefore the fine-structure splittings in the $L S$-multiplets are not yet introduced. However, it is clear from the observed smallness of these splittings and from the arguments given above, that the tensor component of the GBE can play only a minor role. Here we also note that the Yukawa part of the interaction in (3) is only of secondary importance. In fact, the pattern of Fig. 1 could also be described with the $\delta$-part (4) alone (and a slightly modified set of parameters).

It is instructive to learn how the GBE affects the energy levels when it is switched on and its strength (coupling constant) is gradually increased (Fig. 2). Starting out

\footnotetext{
${ }^{1}$ In the meantime the results have been cross-checked with the help of the stochastic variational method [12], and excellent agreement was found.
} 
from the case with confinement only, one observes that the degeneracy of states is removed and the inversion of the ordering of positive- and negative-parity states is achieved, both in the $N$ and $\Delta$ excitations. The reason for this behaviour lies in the flavor dependence of the GBE (see a more detailed discussion in ref. [2]). From Fig. 2 also the crucial importance of the chiral interaction $V_{\chi}$ becomes evident. Notice that the strength of our confinement, $C=0.474 \mathrm{fm}^{-2}$, is rather small and the confining interaction contributes much less to the splittings than the GBE.

We have also calculated the nucleon and delta root mean square "matter" radii, $\sqrt{<} r_{N}^{2}>=0.465 \mathrm{fm}$ and $\sqrt{<} r_{\Delta}^{2}>=0.54 \mathrm{fm}$. For the nucleon one may view this result vis-à-vis the axial r.m.s. radius $\sqrt{<} r_{\text {axial }}^{2}>=0.68(2) \mathrm{fm}$ and also the proton charge r.m.s. radius $\sqrt{<} r_{p}^{2}>=0.862(12)$ fm. It is clear that our result must be smaller, as both of these phenomenological values include (additive and non-additive) effects from the finite size of the constituent quarks and from meson-exchange currents. It is convincing to observe, however, that we obtain $r_{N}<r_{\Delta}$, in line with expectations.

A natural question to be addressed at this point is the role of one-gluon-exchange (OGE) between the constituent quarks [13]. Evidently, OGE alone cannot, in principle, describe the $N$ and $\Delta$ spectra correctly [14]. One may ask, however, if there is an additional contribution of OGE beyond GBE. Such a situation was investigated in a very recent work [15]. There a OGE with a relatively strong coupling constant of $\alpha_{s}=0.7$ was assumed in addition to GBE. A comparison to the results in ref. [15 reveals that our model with GBE alone is capable of describing the $N$ and $\Delta$ spectra in a much better and more comprehensive manner. While the authors of ref. [15] consider only 5 splittings, we are able to reproduce the masses of the 14 lowest $N$ and $\Delta$ states. Clearly, we might include small effects from OGE (i.e. with a rather weak, but realistic, coupling $\alpha_{s}=0.1-0.2$, say) but OGE would then be negligible relative to GBE. Any sizeable coupling $\alpha_{s}>0.2-0.3$ would spoil the spectra, however. This 
is most dramatically seen in the ordering of the positive-parity state $\Delta(1600)$ and the negative-parity $\Delta(1620)-\Delta(1700)$ pair. The color-magnetic interaction shifts the $\Delta(1600)$ strongly up but not down with respect to $\Delta(1620)-\Delta(1700)$ [14]. In addition, the OGE with large $\alpha_{s}$ would imply strong spin-orbit forces while there are practically no spin-orbit splittings in the $N$ and $\Delta$ spectra. Note also that the effect of $\eta^{\prime}$ exchange cannot be generated by the OGE, as the color-magnetic contribution from OGE has an opposite sign.

For the $\eta^{\prime}$-quark coupling $\frac{g_{0}^{2}}{4 \pi}$ we have a value that is bigger than for $\pi$-quark coupling. This is quite natural to expect as the $\eta^{\prime}$ mass is twice larger than the $U(3)$ current algebra prediction (due to the $\mathrm{U}(1)$ anomaly). It is comforting that our value for $\left(g_{0} / g_{8}\right)^{2}$ is within the limits deduced in refs. [16, 17] from the Gottfried sum-rule violation, the $\bar{u} / \bar{d}$ ratio, as well as the flavor and spin contents of the nucleon. The main idea of that work and a previous paper [18] is that the content of the quark sea in the nucleon is governed by the coupling between valence quarks and goldstone bosons.

Our concept of GBE between constituent quarks may also be viewed in the light of some recent evidences from lattice QCD. It was shown that one can obtain a qualitatively correct splitting between $N$ and $\Delta$ already within quenched approximation [19]. However, it was not clear from this result what where the physical reasons for this splitting, OGE, instantons, or something else. To clarify this question, the $N$ - $\Delta$ splitting has recently been measured in the quenched and a further, so-called valence, approximation [20]. While the standard quenched approximation contains part of antiquark effects related to $Z$ graphs formed of valence quark lines, in the valence approximation the quarks are limited to propagate only forward in time (i.e. $Z$ graphs and corresponding $q \bar{q}$ pairs do not enter). The gluon exchange and all other possible gluon configurations, including instantons, are exactly the same in both approximations. The striking result is that the $N-\Delta$ splitting is observed only in 
the quenched approximation but not in the valence approximation, in which the $N$ and the $\Delta$ levels are degenerate within error bars. Consequently the $N-\Delta$ splitting must receive a considerable contribution from $q \bar{q}$ excitations but not from the gluon exchanges or instanton-induced interactions between the constituent quarks.

Summarizing, in this paper we have presented first results for baryon spectra obtained from the GBE model within rigorous 3-particle Faddeev calculations. We relied on an interaction between the constituent quarks stemming from the exchange of octet and singlet pseudoscalar mesons. A simple parametrization of its spin-spin component was enough to achieve the absolute values of the masses of the 14 lowest states in the $N$ and $\Delta$ spectra. The efficiency of the model is evidently due to the spin-flavor dependence introduced by GBE. It leads to an immediate resolution of hitherto persisting problems in baryon spectroscopy. Certainly, the GBE model needs to be extended and refined further. Given the indications of refs. [1, 2] we expect to be able to explain also the strange baryon spectra. For the fine-structure splittings of the LS multiplets and detailed properties of baryon structure, obviously, also the tensor forces have to be included. Corresponding work in both of these directions is presently under way.

The authors are grateful to K. Varga for checking the results with his stochastic variational method. L.Ya.G. is thankful to D.O. Riska for many stimulating discussions and to G.E. Miller for communicating the results of ref. [15]. This work was partially supported by the Austrian-Hungarian Scientific-Technical Cooperation within project A23 (Austria) resp. 17/95 (Hungary). The work of Z.P. was also supported by OTKA grant T17298.

\section{References}

[1] L. Ya. Glozman and D. O. Riska, The Baryon Spectrum and Chiral Dynamics, Preprint HU-TFT-94-47, [LANL hep-ph 9411279] (1994); Systematics of the 
Light and Strange Baryons and the Symmetries of QCD, Preprint HU-TFT-9448, [LANL hep-ph 9412231] (1994).

[2] L. Ya. Glozman and D. O. Riska, The Spectrum of the Nucleons and the Strange Hyperons and Chiral Dynamics, Preprint DOE/ER/40561-187-INT9516-01, [LANL hep-ph 9505422], Phys. Rep. (1996), in print.

[3] G. 't Hooft, Phys. Rev. D14 (1976) 3432.

[4] S. Weinberg, Physica 96 A (1979) 327.

[5] A. Manohar and H. Georgi, Nucl. Phys. B234 (1984) 189.

[6] D. I. Diakonov and V. Yu. Petrov, Phys. Lett. B147 (1984) 351; Nucl. Phys. B272 (1986) 457.

[7] E.V. Shuryak, Phys. Rep. C115 (1984) 152; Rev. Mod. Phys. 65 (1993) 1.

[8] L. Ya. Glozman and D. O. Riska, Quark Model Explanation of the $N^{*} \rightarrow N \eta$ Branching Ratios, Preprint HU-TFT-95-49, [LANL hep-ph 9508327], Phys. Lett. $\mathrm{B}$, in print.

[9] M. Gell-Mann, R. J. Oakes, and B. Renner, Phys. Rev. 175 (1968) 2195.

[10] S. Weinberg, Phys. Rev. D11 (1975) 3583.

[11] Z. Papp and W. Plessas, Coulomb-Sturmian Separable Expansion Approach: Three-Body Faddeev Calculations for Coulomb-Like Interactions, Preprint UNIGRAZ-UTP 26-01-96

[12] K. Varga and Y. Suzuki, Phys. Rev. C52 (1995) 2885; K. Varga, private communication.

[13] A. DeRujula, H. Georgi, and S. L. Glashow, Phys. Rev. D12 (1975) 147.

[14] L. Ya. Glozman, Chiral Symmetry and the Constituent Quark Model, Talk given at the Baryons '95 (7th Int. Conf. on the Structure of Baryons, Santa Fe, October 3-7,1995), [LANL hep-ph 9510440], World Scientific, Singapore, in print.

[15] Z. Dziembowski, M. Fabre de la Ripelle, and G.A. Miller, Non-Perturbative Gluons and Pseudoscalar Mesons in Baryon Spectroscopy, Preprint LANL nuclth 9601022 .

[16] T.P. Cheng and L.-F. Li, Phys. Rev. Lett. 74 (1995) 2872.

[17] T.P. Cheng and L.-F. Li, Antiquark Polarization Inside the Proton Is Small, Preprint LANL hep-ph 9510235, Phys. Lett. B, in print. 
[18] E.J. Eichten, I. Hinchliffe, and C. Quigg, Phys. Rev. D45 (1992) 2269.

[19] D. Weingarten, Nucl. Phys. B (Proc. Suppl.) 34 (1994) 29

[20] K.-F. Liu and S.-J. Dong, Quark Model from Lattice QCD, Preprint UK 194-03, LANL hep-lat 9411067 
Table 1

Masses of the 14 lowest states for non-strange three-quark systems with total orbital angular momentum L, spin $\mathrm{S}$, and isospin $\mathrm{T}$.

\begin{tabular}{|c|c|c|c|}
\hline${ }^{2 T+1} 2 S+1 L$ & Parity & $\begin{array}{l}\text { Theoretical } \\
\text { Mass in MeV }\end{array}$ & $\begin{array}{l}\text { Corresponding phenomenological } \\
\text { LS multiplet }\end{array}$ \\
\hline${ }^{22} S$ & + & 939 & $N$ \\
\hline${ }^{44} S$ & + & 1232 & $\Delta$ \\
\hline${ }^{22} S$ & + & 1493 & $N(1440)$ \\
\hline${ }^{44} S$ & + & 1635 & $\Delta(1600)$ \\
\hline${ }^{22} P$ & - & 1539 & $N(1535)-N(1520)$ \\
\hline${ }^{42} P$ & - & 1667 & $\Delta(1620)-\Delta(1700)$ \\
\hline${ }^{24} P$ & - & 1640 & $N(1650)-N(1700)-N(1675)$ \\
\hline${ }^{22} D$ & + & 1635 & $N(1680)-N(1720)$ \\
\hline${ }^{22} S$ & + & 1690 & $N(1710)$ \\
\hline
\end{tabular}




\section{Figure Captions}

Fig.1

Energy levels for the 14 lowest non-strange baryons with total angular momentum and parity $J^{P}$. The shadowed boxes represent experimental uncertanties.

Fig.2

Level shifts of some lowest baryons as a function of the strength of the GBE. Solid and dashed lines correspond to positive- and negative-parity states, respectively. 


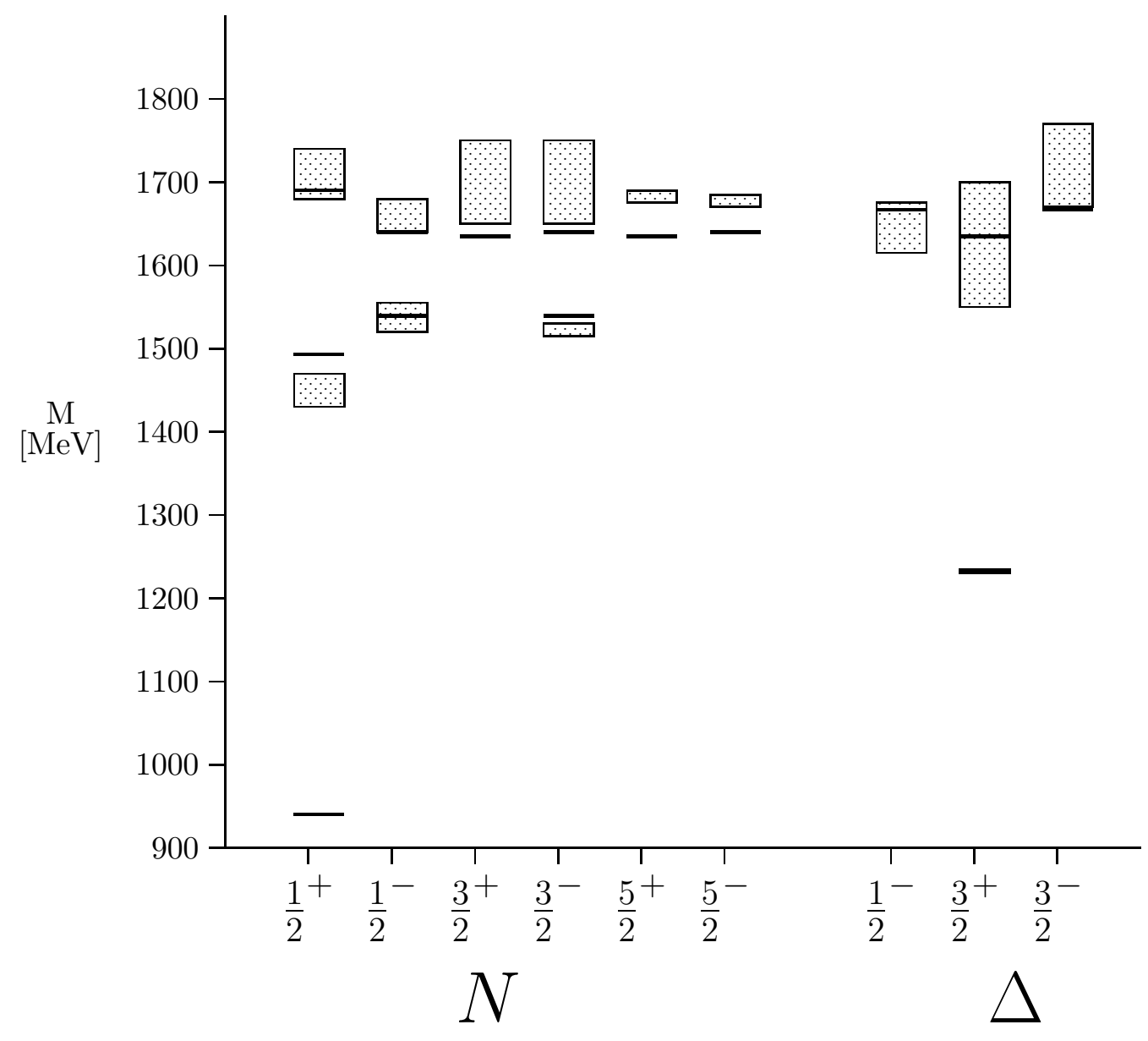

Fig 1: 


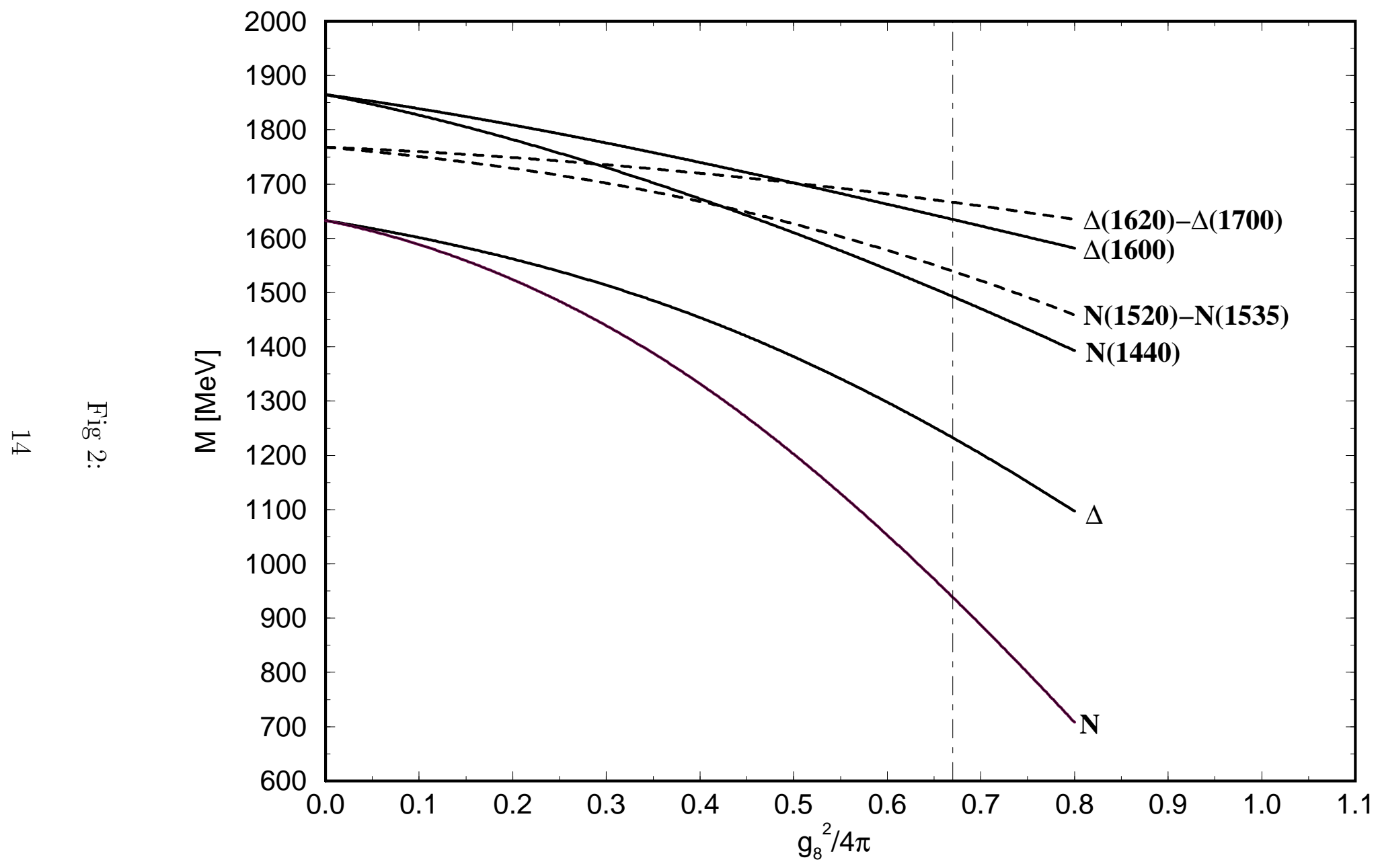

